\title{
II. Hints tending to disprove the existence of distinct calorific rays in the sunbeam
}

\author{
Mr. Henry Meikle
}

To cite this article: Mr. Henry Meikle (1825) II. Hints tending to disprove the existence of distinct calorific rays in the sunbeam , Philosophical Magazine Series 1, 65:321, 10-12, DOI: $10.1080 / 14786442508644648$

To link to this article: http://dx.doi.org/10.1080/14786442508644648

里 Published online: 27 Jul 2009.

Submit your article to this journal $\sqsubset \pi$

Џ Article views: 2

Q View related articles $₫$ 
a function must possess; but it is impossible that one can be found that will accurately represent a probability, of which we never can acquire a perfect knowledge. Therefore, although the same conclusion be still brought out, yet, as the reasoning involves precarious suppositions, the fundamental principles will not be placed in a light so simple and clear and steady as when nothing extraneous is introduced in the demonstration. It has been proved in the case of a great number of observations, that the principle of the least squares will hold good, whatever law of probability be adopted; and hence we may derive an argument that such laws are foreign to the demonstration, since they disappear at the conclusion. If we admit that, whatever be the expression of the chance of an error, the mean of the squares of the errors will converge to a fixed limit as the observations increase in number, the second of the two demonstrations given above will be conclusive in all possible laws of probability.

But, allowing that the method of the least squares is fully established, it is not sufficient for the purposes of astronomy in its present state of improvement. An element, although computed in the best way, can only have a degree of accuracy relative to the precision of the observations. It thus becomes necessary to estimate the uncertainty in the result obtained, or to assign the probability that the remaining error amounts to a tenth, or some given part, of the whole. But the discussion of the subject in this new view of it would exceed our present limits, and must be left to a future occasion.

January 8,1825 .

JAMES Irory.

II. Hints tending to disprove the Existence of distinct Calorific Rays in the Sunbeam. By Mr. Hentr Meikle.

To the Editors of the Philosophical Magazine and Journal. Gentlemen,

TTHAT great mechanical genius Dr. Hooke seems to have 1 been the first who suspected that the sunbeam contains rays which illuminate without producing heat. In this. opinion he has been followed successively by M. Scheele, Sir William Herschel, and Sir Henry Englefield, who are well known as able experimenters. Among other conclusions, the two last thought they had established that the sun emits several distinct sorts of rays, especially illuminating rays, as already mentioned, which give no heat; and on the other hand calorific rays which are not accompanied with light. For on placing a thermometer in the well-known figure called the 
spectrum, this thermometer seemed to be more affected the nearer it was placed to the red margin, and less as it approached the opposite or violet-coloured edge. But the most remarkable effect of all was, that the thermometer indicated the greatest heat when placed just without the red margin, where none of the visible rays reached it at all. They therefore inferred that this effect was produced by a set of dark calorific rays which are less refrangible than any of the other rays. On repeating these experiments, M. Berard obtained similar results, except that he found the maximum of heat in the red ray.

But notwithstanding the high reputation of these philosophers, their conclusion has been questioned by Professor Leslie of Edinburgh, who by experimenting somewhat differently was unable to detach any of these dark rays from the light. Having rendered a circular spot opaque in the middle of a large convex lens, he received the light transmitted by the remaining transparent ring upon a surface of black wax, held at such a distance that the light formed upon the wax an iris, or ring, composed of a set of distinct concentric rings, which severally possessed all the various colours of the common spectrum. Mr. Leslie then carefully observed the effect of these rings on the wax, and found that none of it was melted beyond where it was covered by the iris; whereas if a set of dark calorific rays had existed, these ought to have more thoroughly melted a larger ring than that whereon the light fell; for the dark rays, if less refrangible than light, would have fallen without the margin of the red ring, which includes all the others.

Now, since this experiment of Professor Leslie is of a more simple and decisive cast than any performed by the gentlemen already mentioned, I am much inclined to give it the preference, and to conclude with him that the rays of light only produce or become heat when they themselves disappear. It is also well known that the more perfectly any surface reflects light, the less will that surface itself be heated by the light.

As I do not recollect to have seen any reason given why Sir William Herschel's mode of experimenting ought to have apparently produced the effect he observed, without calling in the aid of dark calorific rays, I shall take the liberty of briefly suggesting what I suspect to have been the principal source of deception. If a prism such as Sir William employed be heated, a very delicate thermometer will, cateris paribus, be' more affected when it is held opposite to one of the flat sides of the prism than when opposite to one of its edges; 
because heat escapes from glass and many other substances, when smooth or polished, chiefly in straight lines.perpendicular to the surface. Now if we attend to the position of Sir William Herschel's prism and thermometer*, this will help to explain why the thermometer indicated heat, even when none of the illuminating rays reached it at all; as also why the heating power of the red rays seemed so much to surpass that of the other colours, \&c.: because the more directly opposite the thermometer was to the flat side of the prism, the more of its heat would it receive; and in the course of such an experiment, there can be little doubt that the prism became considerably heated by absorbing a portion of the solar rays.

The circumstance of heat emerging from an even or polished surface chiefly in perpendicular lines will help to explain various phænomena. From it we learn that Mr. Leslie's lens, however hot, could have little or no tendency to melt the wax; because the heat would emanate from its convex surface chiefly in a set of diverging straight lines, such as might be drawn from the centre of the sphere of which the lens formed a part.

For aught that is known to the contrary, the heating powers of all the rays in the spectrum may be equal; because the seeming difference may be owing to a difference of density, some of them being probably much dilated or attenuated by refraction.

Dec. $21,1824$.

I am, gentlemen, yours \&c.

Henry Meikle.

III. On the Experiments of the Pendulum made by Captain Kater, M. Biot, \&c. By Wm. Galbraith, Esq. A.M.

To the Editors of the Philosophical Magazine and Journal. Gentlemen,

$\mathrm{N}$ my paper published in your Magazine for September 1824, I gave, page 16:, a general formula ( 7 ) to determine the ellipticity of the meridian from observations on the length of the pendulum made in different latitudes. In this formula, however, it is necessary to substitute quantities which, with the exception perhaps of the time of rotation, are not yet sufficiently well determined. The accuracy of these is constantly improving, and in a short time we expect to bave the length of the pendulum oscillating seconds on the equator ascertained by different observers in several points of that circle

* Diagrams or plates illustrative of this may be seen in so many books, that it would be superfluous to introduce them here. 